\title{
Crystallization of Silicon Thin Films by Infrared Semiconductor Laser Irradiation Using Carbon Particles
}

\author{
T. Sameshima ${ }^{*}$, K. Kogure ${ }^{*}$, S. Yoshidomi ${ }^{*}$, T. Haba ${ }^{*}$, M. Hasumi ${ }^{*}$ and N. Sano ${ }^{* *}$ \\ ${ }^{*}$ Tokyo University of Agriculture and Technology, 2-24-16, Naka-cho, Koga nei, Tokyo, 184-8588, Japan \\ Email: tsamesim@cc.tuat.ac.jp \\ ${ }^{* *}$ Hightec Systems Corporation, 3-9-15, Shin Yokohama, Kohoku-ku, Yokohama, Kanagawa, 222-0033, Japan
}

\begin{abstract}
We report the crystallization of 40-nm-thick hydrogenated amorphous silicon films using 940$\mathrm{nm}$ continuous wave infrared semiconductor laser with $200 \mathrm{~nm}$ diameter carbon particles as photo absorption. The laser beam was focused to $100 \mu \mathrm{m}$ diameter at the surface of carbon particles. Laser irradiation at $25 \mathrm{~W}$ heated the carbon surface region to about $3430 \mathrm{~K}$, which was estimated by analysis of spectra of light emission caused by laser irradiation when quartz substrates were placed on the surface of carbon particles. The silicon films coated with $\mathrm{SiO}_{2}$ films were crystallized by laser irradiation for $170 \mu \mathrm{s}$ at $25 \mathrm{~W}$. The crystallization volume ratio was 0.53 . Crystallization over 1.1 $\mathrm{mm}$ area was also demonstrated by laser irradiation at $25 \mathrm{~W}$.
\end{abstract}

DOI: 10.2961/jlmn.2009.03.0016

Keywords: Carbon Optical absorption, Semiconductor laser, Optical reflectivity, Raman scattering

\section{Introduction}

Laser-induced rapid crystallization using pulsed excimer lasers has been widely used for fabricating polycrystalline silicon (poly-Si) films, which have been applied to thin film transistors (TFTs) and solar cells. Laser-induced rapid crystallization has an advantage of a low processing temperature. It allows to fabricate poly-Si TFTs at $300^{\circ} \mathrm{C}$, which is suitable for fabricating switching transistors of pixels and peripheral driver circuits of liquid crystal displays (LCDs) [1-5]. However there have been problems of a low laser power of excimer laser at most 400 $\mathrm{W}$ and high maintenance cost because of toxic gases.

We have developed continuous wave $(\mathrm{CW})$ infrared semiconductor laser annealing using carbon layer as an optical absorption layer.[6-8] Infrared semiconductor lasers with a power of $10 \mathrm{~kW}$ and a high conversion efficiency of $50 \%$ are available. A high power laser is attractive for rapid laser annealing with a short tact time. In order to achieve rapid annealing using $\mathrm{CW}$ laser, we have developed optics shaping a narrow and uniform line laser beam with a length of $15 \mu \mathrm{m}$ and a rapid movement system at $1 \mathrm{~m} / \mathrm{s}$. A laser intensity of $500 \mathrm{~kW} / \mathrm{cm}^{2}$ and $15 \mu \mathrm{s}$ rapid irradiartion have been achieved at the sample surface [9].

In order to solve a problem of low optical absorbance of silicon in the infrared region, we have used carbon as a photo absorption layer [10]. Carbon, especially diamond like carbon (DLC), has outstanding properties of high hardness, good thermal conductivity, wear resistance, thermal durability and chemical inertness. Therefore, it has been widely utilized for technological and industrial applications such as wear resistant and protective hard coats. DLC has also interesting optical properties, which are low refractive indices from 1.3 to 1.9 and high extinction coefficients from 0.8 to 0.9 for wavelengths from 250 to $1100 \mathrm{~nm}$. When carbon films are formed on siliocn, laser diodes with wavelengths of $800-1000 \mathrm{~nm}$ can be used for the crystallization of silicon films. Carbon films absorb laser light and heat themselves to a high temperature and also heat adjacient silicon films by heat diffusion. At present sputtering mtehod for carbon film formation has been well established in the industry field. Moreover, it is necesarry to look for a further possibility of crystallization process using a combination of infrared laser with carbon for low cost processing.

In this paper, we report crystallization of silicon films using carbon particles. We report heating properties to a high temeprature about $3400 \mathrm{~K}$ of carbon particles by laser irradiation by observing light emission from carbon particles. We also discuss crystallization conditions of the laser power and the laser dwell time. We also report crystalline properties including crystalline volume ratio using Raman scattering method.

\section{Experimental Details}

Figure 1(a) shows an experimental apparatus. An infrared semiconductor laser beam with a wavelength of $940 \mathrm{~nm}$ and a power of $25 \mathrm{~W}$ was introduced by an optical fiber. The optical fiber and optics with lens were mounted on the $\mathrm{X}-\mathrm{Y}$ mobile stage. The laser was moved at $1 \mathrm{~m} / \mathrm{s}$ at maximum in the X-direction. It was also moved in the Ydirection with a step. Laser light was focused by lens to a spot with a Gaussian-like intensity distribution with a diameter of $100 \mu \mathrm{m}$. A stainless-steel chamber with a diameter of $6 \mathrm{~cm}$ and a depth of $1 \mathrm{~cm}$ was filled with carbon particles with a mean diameter of $200 \mathrm{~nm}$, as shown in Fig. 1(b). The top surface of samples was carefully placed on the surface of carbon particles by a mechanically stress with no substantial air gaps. The samples were irradiated with $940 \mathrm{~nm}$ infrared semiconductor laser beam from the rear side of the transparent substrates. Carbon particles absorbed laser light and heated themselves. The heating energy propagated to the samples and silicon films were heated by the heat diffusion. Carbon particles can effectively absorb the infrared light because the optical reflectivity at the surface of carbon surface was low about $10 \%$ due to 


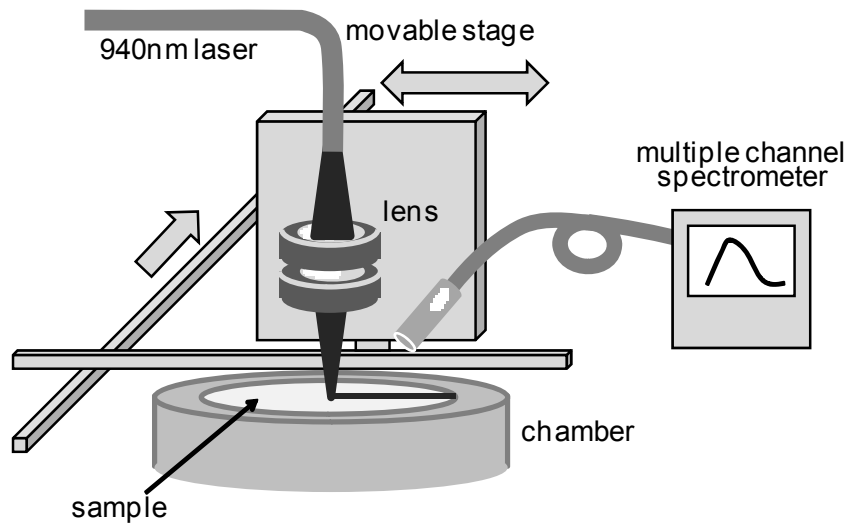

(a)
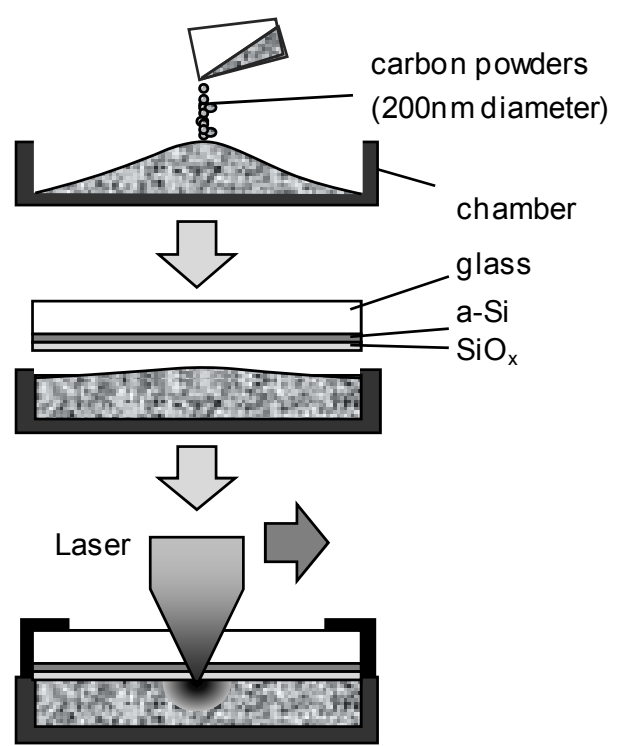

(b)

Fig.1: Experimental apparatus with a $940 \mathrm{~nm}$ infrared semiconductor laser (a) and image of laser annealing of samples using carbon particles as photo-absorption.

a low refractive index [10]. Moreover, there is light scatter scattering in the case of carbon particles. However, there was a reflection loss at the quartz glass/Si layer interface when the laser light was irradiated from the rear side. Because the reflectivity was $35 \%$ at $940 \mathrm{~nm}$ for quartz glass/40-nm-a-Si layer, we interpret the optical absorption efficiency was about $60 \%$ for the present experimental system.

In order to estimate temperature increase of carbon particles by laser heating, light emission from carbon surface was observed using multichannel spectrometer from 250 to $750 \mathrm{~nm}$, as shown in Fig. 1(a). Quartz substrates were placed on the surface of carbon particles. Light emission from quartz/carbon interfaces was measured using spectrometer during laser irradiation with different laser powers ranging from 7 to $25 \mathrm{~W}$. The sensitivity of the detector with different wavelengths was calibrated using a black body furnace. The emission spectra was analyzed by black body radiation model using Planck's radiation equation, I=
$8 \pi h c \lambda^{-5}[\exp (h c / \lambda k T)-1]^{-1}$, where $h$ is Planck's constant, $c$ is the light velocity, $\lambda$ is the wavelength, $k$ is the Boltzmann's constant and $T$ is absolute temperature. The emissivity of carbon particles was supposed to be 1 . For experiment of laser crystallization, 40-nm-thick undoped hydrogenated amorphous silicon (a-Si:H) films with a hydrogen concentration of $20 \%$ were formed on quartz glass substrates by plasma enhanced chemical vapor deposition. 200-nm-thick $\mathrm{SiO}_{\mathrm{x}}$ films were subsequently formed on the a-Si:H films using Polysilazane liquid. The samples were then heated at $260^{\circ} \mathrm{C}$ with $1.3 \times 10^{6} \mathrm{~Pa} \mathrm{H}_{2} \mathrm{O}$ vapor for $3 \mathrm{~h}$ for formation of $\mathrm{SiO}_{2}$ films from Polysilazane layers.

\section{Results and discussions}

Figure 2 shows spectra of light emission with different laser powers (a) when the infrared laser was focused to the surface of carbon particles though a quartz substrate for $1 \mathrm{~s}$, and temperature as a function of laser power (b). The emissions showed broad spectra and the emission intensity increased as the wavelength increased. It also increased as the laser power increased. The emission spectra were analyzed by the black body radiation theory using Planck's radiation equation. The fitting calculated spectra to experimental ones shown in Fig. 2(a) gave most possible temperature as a function of laser power, as shown in Fig. 2 (b).The temperature increased from 2750 to $3050 \mathrm{~K}$ as laser

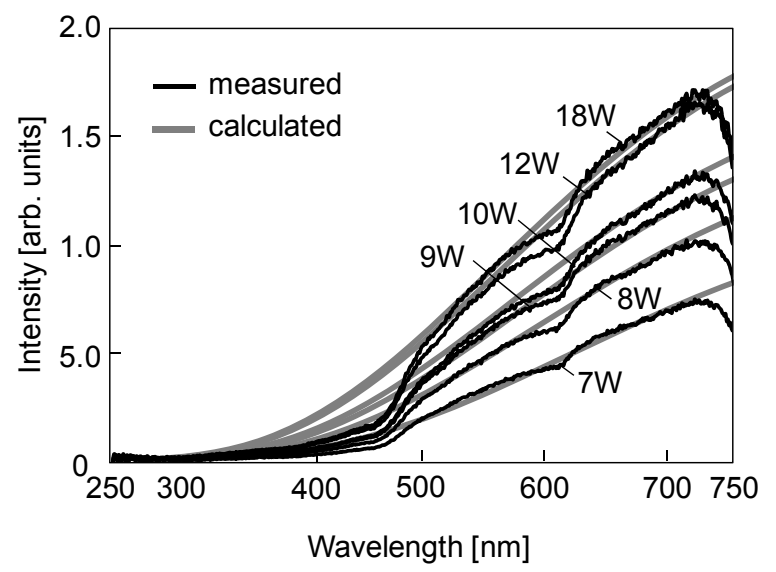

(a)

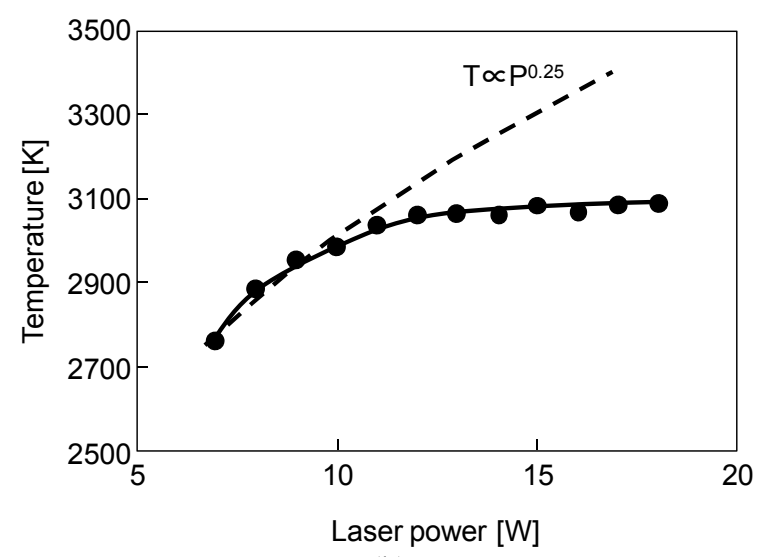

(b) 
Fig.2: light emission spectra for laser irradiation for $1 \mathrm{~s}$ with different laser powers (a), and temperature as a function of laser power (b)

power increased from 7 to $11 \mathrm{~W}$. It is important that carbon particles were heated to temperature higher than the melting point of silicon $(1680 \mathrm{~K})$. Behavior of temperature increase was almost proportional to laser power to the power of one fourth. It means that light emission occurred according to Stefan-Boltzmann law. On the other hand, temperature almost leveled off at $3050 \mathrm{~K}$ for laser power above 12 W. This saturation was probably caused by substantial dissipation of heating energy through explosively movement of carbon particles or distortion of glass substrate. Small holes at the carbon surface and small grooves at the surface of quartz substrate were observed by naked eyes after laser irradiation above $16 \mathrm{~W}$.

Figure 3 shows temperature as a function of laser dwell time when the infrared laser was moved at velocities ranging from 10 to $100 \mathrm{~cm} / \mathrm{s}$ at different powers of 10,18 and $25 \mathrm{~W}$. Temperature slightly increased as the laser dwell time increased from 100 to $500 \mu$ s for every laser power. The maximum temperatures of 2960, 3200 and $3430 \mathrm{~K}$ were obtained for the cases of $10,18,25 \mathrm{~W}$ irradiation, respectively. The maximum temperatures for 18 and $25 \mathrm{~W}$ were higher than the maximum temperature, $3050 \mathrm{~K}$, for the case of stationary irradiation for $1 \mathrm{~s}$. No damage was observed in the carbon particles and quartz substrate. Carbon particles did not substantially moved and heating energy was effectively kept. Solid curves were temperatures calculated including heat energy losses by black body radiation and heat diffusion. In the short laser dwell time lower than $200 \mu \mathrm{s}$, heat diffusion was important and temperature was decreased by heat conduction to carbon and glass substrate. On the other hand, the highest temperature was almost governed by radiation energy loss in the case of

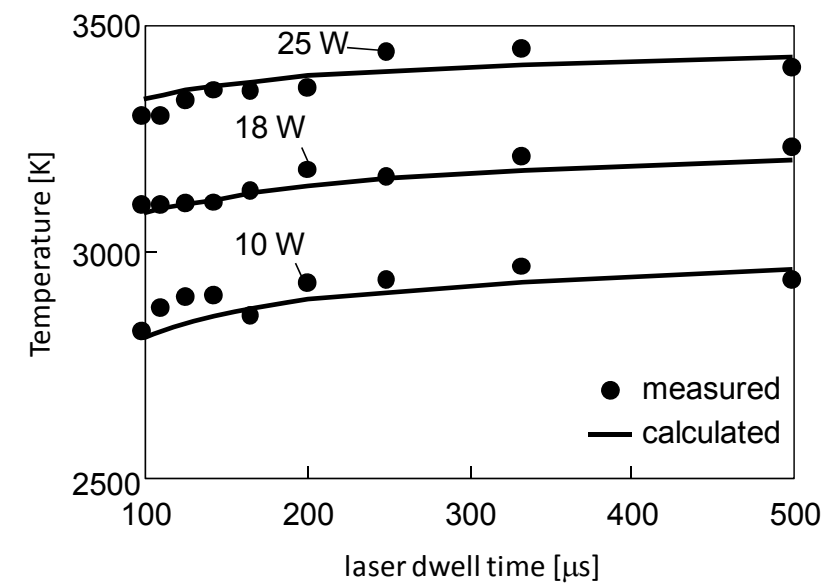

the laser dwell time longer than $200 \mu$ s because of spatial limitation of heat conduction.

Fig.3: Temperature as a function of laser dwell time when the infrared laser was moved at velocities ranging from 10 to $100 \mathrm{~cm} / \mathrm{s}$ at different powers.

Figure 4 shows Raman scattering spectra with different dwell times when the laser beams at $25 \mathrm{~W}$ were irradiated and moved one way. The color of the silicon films were changed by laser irradiated for line-shape regions with a width of about $100 \mu \mathrm{m}$ as shown by inset of Fig.4. Raman scattering was measured at the middle points of laser irradiated regions. The crystalline silicon phonon peaks appeared around wavenumber of $519 \mathrm{~cm}^{-1}$ for a laser dwell time of $143 \mu \mathrm{s}$. The intensity of crystalline silicon phonon peak increased as the laser dwell time increased. The silicon films were crystallized by heat diffusion from carbon particles heated by laser irradiation.

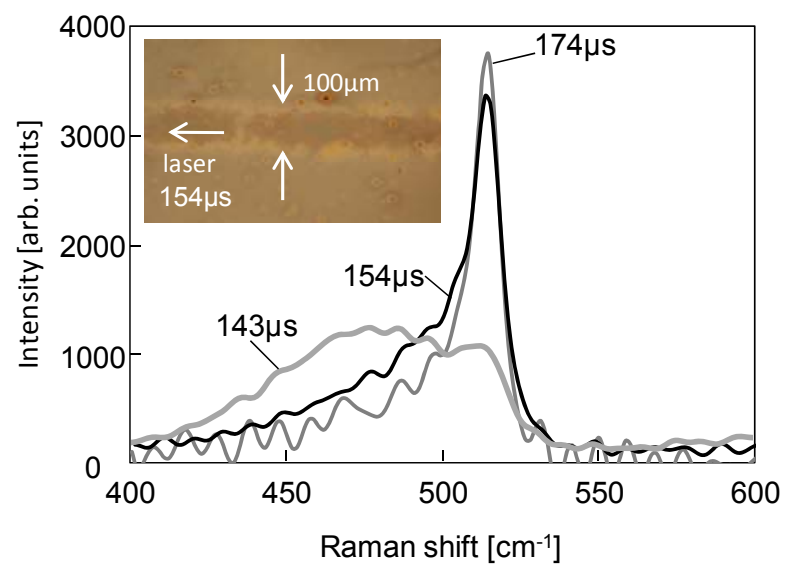

Fig.4: Raman scattering spectra with different dwell times when the laser beam at $25 \mathrm{~W}$ was irradiated and moved one way. Inset represents a photo of silicon surface laser irradiated for $154 \mu \mathrm{s}$.

Figure 5 shows crystalline volume ratio as a function of the laser dwell time when laser beam at 18 and $25 \mathrm{~W}$ was irradiated to samples. The crystalline volume ratio was obtained by analysis of Raman scattering spectra assumed by three phonon bands of crystalline at $519 \mathrm{~cm}^{-1}$, nano-crystalline at $500 \mathrm{~cm}^{-1}$ and amorphous at $480 \mathrm{~cm}^{-1}$. It was estimated intensity ratio of the crystalline band to those of total three bands. The crystalline volume ratio increased from 0.03 to 0.53 as the laser dwell time increased from 143 to $175 \mu \mathrm{s}$ in the case of $25 \mathrm{~W}$. Laser irradiation with a high laser intensity $2.1 \times 10^{5} \mathrm{~W} / \mathrm{cm}^{2}$ ( $25 \mathrm{~W}$ case) heated carbon particles to a high temperature and achieved crystallization of silicon with a short dwell time. Surface roughness was observed in the case of laser irradiation longer than $180 \mu \mathrm{s}$. It probably resulted from that silicon films were melted and significant movement of silicon atoms occurred on the glass substrate. On the other hand, a long heating duration of $440 \mu$ s was necessary to crystallize silicon films at $18 \mathrm{~W}$. It indicates that heating carbon to very high temperature is important to crystallize silicon films by heat conduction from carbon particles.

Figure.6 shows crystalline volume ratio distribution over $1.1 \mathrm{~mm}$ measured in the normal direction of laser scanning. $1.1 \mathrm{~mm}$ region was irradiated by laser beam at $25 \mathrm{~W}$. Laser irradiation was repeated in the plus or minus at $60 \mathrm{~cm} / \mathrm{s}$ in the $\mathrm{X}$ direction with a dwell time of $150 \mu \mathrm{s}$. It paused at edges a little before moving again in the opposite direction. During the pause, the laser beam was moved stepwise by $50 \mu \mathrm{m}$ in the Y direction. Overlapping 
rate was $50 \%$. The average crystalline volume ratio was 0.45 . The crystalline volume ratio distributed from 0.3 to 0.58 . While this demonstration shows a possibility of

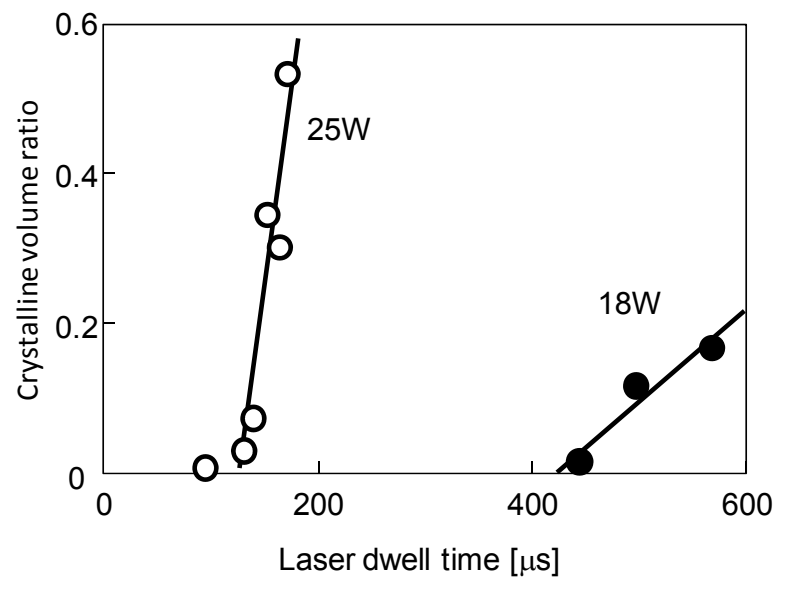

Fig.5: Crystalline volume ratio as a function of the laser dwell time with different laser powers of 18 and $25 \mathrm{~W}$.

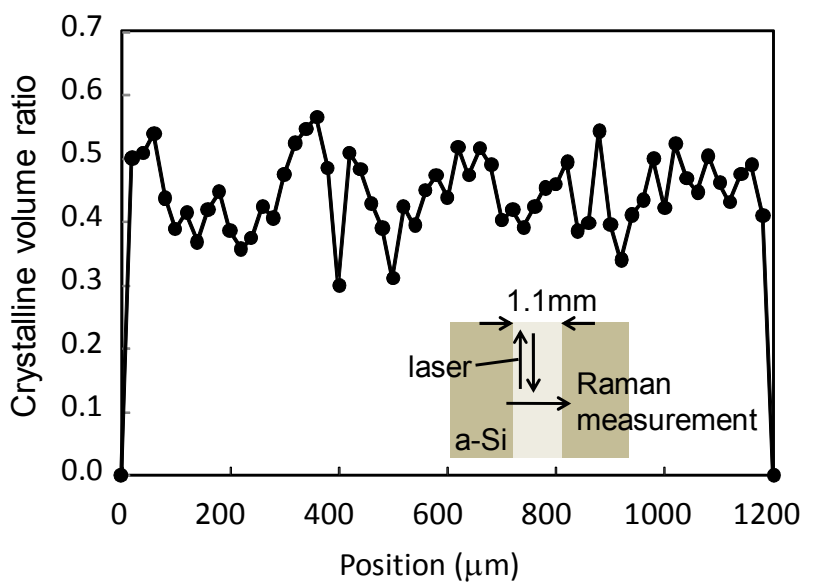

Fig.6: Crystalline volume ratio distribution over $1.1 \mathrm{~mm}$ measured in the normal direction of laser scanning region at $25 \mathrm{~W}$ as shown in inset.

crystallization of silicon film over a large area, there is a problem in uniformity of the crystalline volume ratio. Carbon contact to silicon should be further improved.

\section{Summary}

Rapid thermal crystallization of silicon films by $\mathrm{CW}$ infrared semiconductor laser irradiation using carbon particles as photo absorption was investigated. A stainless-steel chamber was filled with carbon particles with a mean diameter of $200 \mathrm{~nm}$. Quartz substrates were placed on carbon particles and irradiated with $940 \mathrm{~nm}$ infrared semicon ductor laser at a diameter of $100 \mu \mathrm{m}$. During irradiation and movement of laser, spectra of light emission from the carbon surface were observed using multichannel spectrometer from 250 to $750 \mathrm{~nm}$. Temperature of carbon particles were estimated by fitting spectra. The temperature increased to $3430 \mathrm{~K}$ at a laser power of $25 \mathrm{~W} .40 \mathrm{~nm}$ thick undoped hydrogenated amorphous silicon (a-Si:H) films were formed on quartz glass substrates by plasma enhanced chemical vapor deposition. $200 \mathrm{~nm}$ thick $\mathrm{SiO}_{\mathrm{x}}$ films were subsequently formed on the a-Si:H films using Polysilazane precursor heated at $260^{\circ} \mathrm{C}$ with $1.3 \times 10^{6} \mathrm{~Pa} \mathrm{H}_{2} \mathrm{O}$ vapor for $3 \mathrm{~h}$. The top surface of silicon films was contacted to carbon particles. The samples were irradiated by laser from the rear side of the transparent substrates. Raman scattering spectra were measured in order to investigate crystallization of silicon films. Crystallization of silicon films were confirmed at dwell time longer than $143 \mu$ s for a laser power of $25 \mathrm{~W}$. The crystalline volume ratio was 0.53 in the case of $170 \mu \mathrm{s}$ dwell time. This result shows that carbon particles can play a role of a good heating source for crystallizing silicon films.

\section{Acknowledgments}

This work was partially supported by NEDO foundation P07026.

\section{References}

[1] T. Sameshima, S. Usui, M. Sekiya: IEEE Electron Dev. Lett. 7 (1986) 276.

[2] A. Kohno, T. Sameshima, N. Sano, M. Sekiya and M. Hara: IEEE Trans. Electron Devices, 42 (1995) 251.

[3] T. Serikawa, S. Shirai, A. Okamoto and S. Suyama: Jpn. J. Appl. Phys. 28 (1989) 1871.

[4] S. Uchikoga and N. Ibaraki: Thin Solid Films, 383 (2001) 19.

[5] T. Shimoda: Proc. of Workshop on Active Matrix Liquid Crystal Displays, (Tokyo, 2002) 5.

[6] T. Sameshima, M. Maki, M. Takiuchi, N. Andoh, N. Sano, Y. Matsuda and Y. Andoh, Jpn. J.Appl. Phys. 46 (2007) 6474.

[7] N. Sano, M. Maki, N. Andoh and T. Sameshima: Jpn. J. Appl. Phys. 46 (2007) 1254.

[8] N. Sano, M. Maki, N. Andoh and T. Sameshima, Y. Matsuda, and Y. Andoh, Jpn. J.Appl. Phys. 46 (2007) L620.

[9] K. Ukawa, T. Sameshima, N. Sano, M. Naito and N. Hamamoto, Proc. in Workshop on Active Matrix Flat Panel Displays (Nara, 2009) 133.

[10] T. Sameshima and N. Andoh: Jpn. J.Appl. Phys. 44 (2005)7305.

(Received: July 10, 2009, Accepted: December 1, 2009) 